\title{
Validation of the Dementia Care Assessment Packet-Instrumental Activities of Daily Living
}

\author{
Seok Bum Lee', Jeong Ran Park', Jeong-Hwa Yoo', Joon Hyuk Park², Jung Jae Lee ${ }^{3}$, \\ Jong Chul Yoon ${ }^{4}$, Jin Hyeong Jhoo ${ }^{5}$, Dong Young Lee ${ }^{6,7}$, Jong Inn Woo ${ }^{6,7}$, Ji Won Han ${ }^{3}$, \\ Yoonseok $\mathrm{Huh}^{3}$, Tae Hui Kim ${ }^{3}$ and Ki Woong Kim ${ }^{3,7} \bowtie$ \\ 1'Department of Psychiatry, Dankook University Hospital, Cheonan, Republic of Korea \\ ${ }^{2}$ Department of Neuropsychiatry, Jeju National University Hospital, Jeju, Republic of Korea \\ ${ }^{3}$ Department of Neuropsychiatry, Seoul National University Bundang Hospital, Seongnam, Republic of Korea \\ ${ }^{4}$ Department of Neuropsychiatry, Kyunggi Provincial Hospital for the Elderly, Yongin, Republic of Korea \\ ${ }^{5}$ Department of Psychiatry, Kangwon National University School of Medicine, Chuncheon, Republic of Korea \\ ${ }^{6}$ Department of Neuropsychiatry, Seoul National University Hospital, Seoul, Republic of Korea \\ ${ }^{7}$ Department of Psychiatry, Seoul National University College of Medicine, Seoul, Republic of Korea
}

Objective We aimed to evaluate the psychometric properties of the IADL measure included in the Dementia Care Assessment Packet (DCAP-IADL) in dementia patients.

Methods The study involved 112 dementia patients and 546 controls. The DCAP-IADL was scored in two ways: observed score (OS) and predicted score (PS). The reliability of the DCAP-IADL was evaluated by testing its internal consistency, inter-rater reliability and test-retest reliability. Discriminant validity was evaluated by comparing the mean OS and PS between dementia patients and controls by ANCOVA. Pearson or Spearman correlation analysis was performed with other instruments to assess concurrent validity. Receiver operating characteristics curve analysis was performed to examine diagnostic accuracy.

Results Chronbach's a coefficients of the DCAP-IADL were above 0.7. The values in dementia patients were much higher (OS=0.917, PS=0.927), indicating excellent degrees of internal consistency. Inter-rater reliabilities and test-retest reliabilities were statistically significant $(\mathrm{p}<0.05)$. PS exhibited higher reliabilities than OS. The mean OS and PS of dementia patients were significantly higher than those of the non-demented group after controlling for age, sex and education level. The DCAP-IADL was significantly correlated with other IADL instruments and MMSE-KC $(\mathrm{p}<0.001)$. Areas under the curves of the DCAP-IADL were above 0.9.

Conclusion The DCAP-IADL is a reliable and valid instrument for evaluating instrumental ability of daily living for the elderly, and may also be useful for screening dementia. Moreover, administering PS may enable the DCAP-IADL to overcome the differences in gender, culture and life style that hinders accurate evaluation of the elderly in previous IADL instruments.

Psychiatry Investig 2013;10:238-245

Key Words Dementia, Instrumental activities of daily living, DCAP.

\section{INTRODUCTION}

The decline of functional activities is the critical feature in the diagnosis of dementia. ${ }^{1}$ Functional assessment instru-

Received: October 5, 2012 Revised: March 20, 2013

Accepted: April 2, 2013 Available online: September 16, 2013

$\triangle$ Correspondence: Ki Woong Kim, MD, PhD

Department of Neuropsychiatry, Seoul National University Bundang Hospital, 166 Gumi-ro, Bundang-gu, Seongnam 463-707, Republic of Korea Tel: +82-31-787-7439, Fax: +82-31-787-4058, E-mail: kwkimmd@snu.ac.kr

(a) This is an Open Access article distributed under the terms of the Creative Commons Attribution Non-Commercial License (http://creativecommons.org/licenses/by$\mathrm{nc} / 3.0$ ) which permits unrestricted non-commercial use, distribution, and reproduction in any medium, provided the original work is properly cited. ments measure activities of daily living (ADL), a measure of a person's ability to live independently. Impaired ADL can be disclosed by clinical interviews. Patients or their families complain about forgetting appointments, difficulties in managing money or dressing themselves, inability to use telephone and so on. ADL assessment is also an important outcome measure in evaluating efficacy of anti-dementia drugs. Along with cognitive measures, ADL measures are commonly included in clinical trials of anti-dementia drugs as a primary outcome measure..$^{2-4}$

ADL can be divided into basic ADL (BADL) and instrumental ADL (IADL). BADL include self-maintenance skills 
such as bathing, dressing and toileting. IADL refers to daily activities that are not necessary for fundamental functioning, but enable the individual to live independently within a community (light housework, preparing meals, taking medications, shopping, using the telephone, managing money and so on). ${ }^{5}$ IADL, in particular, requires greater complexity of neuropsychological organization. IADL is more likely to be vulnerable to cognitive decline, ${ }^{5-9}$ and thus its impairment may be an important indicator of early dementia. ${ }^{2,3,10-12}$ Some studies showed that IADL measures are useful screening instruments for dementia. ${ }^{13-15}$ In addition, IADL can also reflect the level of dependency and prognosis of dementia patients. ${ }^{2-4}$

IADL can be measured in three ways: self-reported questionnaires, performance-based assessment and informantbased questionnaires. ${ }^{16}$ Self-reported questionnaires are difficult to accomplish for dementia patients since most dementia patients can not aware their functional impairment. ${ }^{17-20} \mathrm{Ob}$ servation or direct assessment has the advantage of directly obtaining information without relying on self- or informantreport. Nonetheless, the major drawback of this method is the time-consuming aspect of these instruments. ${ }^{2,21}$ Hence, the most common method for assessing dementia is the informant-based questionnaires. Currently, various informantbased questionnaires for IADL are available and their number is still growing. ${ }^{2,22}$ Also, a number of Korean instruments are available for IADL. ${ }^{23-25}$

Despite the widespread use of these assessment tools, there are some limitations in the current measures of IADL. First, some measures include items that are relatively unrelated to IADL (memory, comprehension, or social functioning). ${ }^{24,25}$ Second, some include items that are not easily applicable to men (for example, grooming). ${ }^{23-25}$ Third, some include both IADL and BADL items together. ${ }^{16}$ Finally, most scales do not score the items that the subject have not experienced within an observation window. This scoring system may make pose difficulties in comparing IADL directly between and within subjects, and result in underestimation of potential functional ability if subjects pursuit a simple lifestyle.

To overcome these limitations in the currently available IADL measures, the IADL measure included in the Dementia Care Assessment Packet (DCAP-IADL) ${ }^{26}$ included only IADL items that are equally applicable to both genders and asked to score not only the items that subjects experienced but also the items that subjects have not experienced within an observation window. In the present study, we evaluated the psychometric properties and usefulness of the DCAP-IADL in evaluating dementia patients.

\section{METHODS}

\section{Subjects}

The participants consisted of 112 dementia patients (79 Alzheimer's disease, 25 vascular dementia, 1 frontotemporal dementia, 4 dementia of Lewy body disease, 4 Parkinson's disease dementia, and 3 other dementia) and 546 non-demented elderly controls aged 60 years or older. The non-demented elderly subjects were community-dwelling Korean elders who participated in the Korean Longitudinal Study for Health and Aging (KLoSHA). ${ }^{27}$ The dementia patients were recruited from either the visitors to the Dementia Special Clinic of Dankook University Hospital $(n=58)$ or the participants of the KLoSHA ( $n=54)$. All subjects were evaluated according to the clinical assessment protocol of the Korean version of the Consortium to Establish a Registry for Alzheimer's Disease Assessment battery (CERAD-K). ${ }^{28}$ The diagnosis of dementia was made according to the DSM-IV diagnostic criteria. ${ }^{29}$ All dementia patients had reliable informants who could provide adequate clinical histories. Among the dementia patients, 27 (24.1\%), $42(37.5 \%)$ and $43(38.4 \%)$ were very mild (CDR= $0.5)$, mild $(\mathrm{CDR}=1)$ and moderate to severe $(\mathrm{CDR}=2$ or 3$)$, respectively.

The demographic characteristics of the subjects are presented in Table 1 . The dementia patients were older $(p<0.001)$ and less educated $(\mathrm{p}<0.001)$ than the non-demented controls. Women were more common in the dementia group than in

Table 1. Demographic characteristics of non-demented control subjects and dementia patients

\begin{tabular}{|c|c|c|c|}
\hline & $\begin{array}{l}\text { Non-demented } \\
\text { control subjects } \\
\quad(\mathrm{N}=546)\end{array}$ & $\begin{array}{c}\text { Dementia } \\
\text { patients } \\
(\mathrm{N}=112) \\
\end{array}$ & $\begin{array}{c}\text { Total } \\
(\mathrm{N}=658)\end{array}$ \\
\hline \multicolumn{4}{|l|}{ Age (years) } \\
\hline Mean \pm SD & $77.65 \pm 8.62$ & $81.54 \pm 8.72$ & $78.31 \pm 8.75$ \\
\hline Range & $65-96$ & $60-98$ & $60-98$ \\
\hline \multicolumn{4}{|l|}{ Sex } \\
\hline Male & $275(50.4)$ & $33(29.5)$ & $308(46.8)$ \\
\hline Female & $271(49.6)$ & $79(70.5)$ & $350(53.2)$ \\
\hline \multicolumn{4}{|c|}{ Education (years) } \\
\hline Mean \pm SD & $7.30 \pm 5.66$ & $4.11 \pm 4.94$ & $6.77 \pm 5.67$ \\
\hline Range & $0-20$ & $0-16$ & $0-20$ \\
\hline \multicolumn{4}{|l|}{ CDR } \\
\hline 0 & $323(59.2)$ & & $323(49.1)$ \\
\hline 0.5 & $223(40.8)$ & $27(24.1)$ & $250(38.0)$ \\
\hline 1 & & $42(37.5)$ & $42(6.4)$ \\
\hline$\geq 2$ & & $43(38.4)$ & $43(6.5)$ \\
\hline
\end{tabular}

Number (percent) of subjects unless otherwise indicated. CDR: Clinical Dementia Rating Scale 
the non-demented control group $(\mathrm{p}<0.001)$.

\section{DCAP-IADL}

The DCAP-IADL consists of a total of 10 items that include 3 community items (mode of transportation, going outside within walking distance, and shopping), 5 personal management items (ability to use the telephone, ability to use electric appliances, ability to handle money, ability to manage personal belongings, and being responsible for own medication), and, 2 household items (housekeeping and food preparation). "Laundry", although it is an important and common activity of daily living, is not included since it is barely performed by men in Asian cultures. All items are rated based on observations in the recent 90 days. Each item is rated from 0 to 3 points. The total score of the DCAP-IADL is 30 points, and a higher score indicates lower instrumental activities of daily living. We scored the DCAP-IADL in two ways: observed score (OS) and predicted score (PS). For scoring OS, informants were asked to score each item based on observations in the recent 90 days. If an item could not be observed during this time period, it was scored as 3 points, which is equivalent to "cannot be carried out". However, in scoring PS, informants were asked to give their best guesses on the items that they have not experienced instead of giving 3 points. For instance, if a female subject did not manage financial matters because her husband takes exclusive charge of handling finance, we requested her informant to predict whether she would be able to manage financial matters if her husband did not take on the job.

\section{Reliability}

Three aspects of reliability were examined internal consistency of scale, inter-rater reliability and test-retest reliability. Internal consistency was examined by Crohnbach's alpha. In order to evaluate inter-rater reliability, 36 dementia patients and 8 non-dementia controls were rated with the DCAPIADL simultaneously by two independent researchers (tester and rater). The DCAP-IADL was administered again to 17 dementia patients and 13 non-dementia controls by the same rater at 2-4 weeks after the initial assessment. For evaluating inter-rater and test-retest reliabilities, Pearson correlation coefficients were calculated.

\section{Validity}

To evaluate discriminant validity, the mean OS and PS were compared between the dementia patients and non-demented subjects by ANCOVA after adjusting for age, sex and educational level. We evaluated the concurrent validity of the DCAP-IADL by calculating its Pearson correlation coefficients with K-IADL, ${ }^{30} \mathrm{BDS}-\mathrm{ADL},{ }^{28}$ Korean version of Mini Mental Status Examination (MMSE-KC) ${ }^{28}$ and the sum of the box scores of the Clinical Dementia Rating (CDR-SOB) $)^{31}$ and a Spearman correlation coefficient with CDR.

To examine diagnostic accuracy, we performed receiver operating characteristics (ROC) curve analysis for both OS and PS of the DCAP-IADL and compared their areas under the ROC curves (AUC) with those of K-IADL and MMSEKC. ${ }^{32}$

All statistical analyses were performed using SPSS for windows, version 13.0 and Medcalc for windows, version 12.2.1.0 (MedCalc Software, Mariakerke, Belgium).

\section{RESULTS}

\section{Reliability}

The results of the reliability analysis are presented in Table 2 . Chronbach's coefficients alpha for OS and PS in non-demented control subjects were both above 0.7 (OS=0.783, $\mathrm{PS}=0.829$ ). The values in dementia patients were much higher ( $\mathrm{OS}=0.917$, $\mathrm{PS}=0.927$ ), indicating excellent degrees of internal consistency. ${ }^{33}$ Inter-rater reliabilities for OS and PS in the non-demented control subjects, as measured by Pearson correlation coefficients, were both statistically significant $(\mathrm{OS}=0.720$, PS= $0.778, \mathrm{p}<0.05)$. The values in dementia patients were much better $(\mathrm{OS}=0.973, \mathrm{PS}=0.968, \mathrm{p}<0.05)$. Test-retest reliabilities for OS and PS in the non-demented control subjects were both statistically significant (OS=0.942, $\mathrm{PS}=0.973, \mathrm{p}<0.05)$. Those in dementia patients were both statistically significant (OS=0.693, $\mathrm{PS}=0.717, \mathrm{p}<0.05$ ), however lower than those in the non-demented controls. Internal consistency and test-re-

Table 2. Results of reliability test for non-demented control subjects and dementia patients

\begin{tabular}{|c|c|c|c|c|}
\hline & \multicolumn{2}{|c|}{ Non-demented control subjects } & \multicolumn{2}{|c|}{ Dementia patients } \\
\hline & $\begin{array}{l}\text { DCAP-IADL } \\
\text { observed score }\end{array}$ & $\begin{array}{l}\text { DCAP-IADL } \\
\text { predicted score }\end{array}$ & $\begin{array}{l}\text { DCAP-IADL } \\
\text { observed score }\end{array}$ & $\begin{array}{l}\text { DCAP-IADL } \\
\text { predicted score }\end{array}$ \\
\hline IC & 0.783 & 0.829 & 0.917 & 0.927 \\
\hline $\mathrm{IR}^{*}$ & 0.720 & 0.778 & 0.973 & 0.968 \\
\hline $\mathrm{TR}^{*}$ & 0.942 & 0.973 & 0.693 & 0.717 \\
\hline
\end{tabular}

*all correlations are significant at 0.05 level by Pearson correlation analysis. IC: internal consistency, IR: inter-rater reliability, TR: test-retest reliability, DCAP-IADL: the Dementia Care Assessment Packet-Instrumental Activities of Daily Living 
test reliabilities for PS of the DCAP-IADL in the non-dementia subjects and the dementia patients were higher than those for OS. Inter-rater reliabilities for PS of the DCAP-IADL in the non-dementia subjects were higher than those for OS, and inter-rater reliabilities for PS of the DCAP-IADL in the dementia patients were comparable to those for OS. As a whole, PS of the DCAP-IADL exhibited higher reliabilities than OS.

\section{Validity}

As shown in Table 3, the DCAP-IADL clearly discriminated the dementia group from the non-demented group. The mean OS and PS of dementia patients were significantly higher than those of the non-demented group after controlling for age, sex and education level ( $\mathrm{p}<0.0001$, ANCOVA). The mean scores of $\mathrm{OS}(\mathrm{df}=4, \mathrm{~F}=266.55, \mathrm{p}<0.0001)$ and $\mathrm{PS}(\mathrm{df}=4, \mathrm{~F}=$ $314.74, \mathrm{p}<0.0001$ ) of the DCAP-IADL for the 3 different dementia stages according to CDR indices were significantly different (Figure 1). In post-hoc multiple comparison analyses, all of the different dementia stages differed significantly from one another $(\mathrm{p}<0.005$, Bonferroni comparison). As summarized in Table 4, OS and PS of the DCAP-IADL were significantly correlated not only with other functional measures such as K-IADL (OS=0.840, PS=0.845, $\mathrm{p}<0.001)$, BDS$\mathrm{ADL}(\mathrm{OS}=0.784, \mathrm{PS}=0.815, \mathrm{p}<0.001)$, and $\mathrm{CDR}-\mathrm{SOB}(\mathrm{OS}=$ $0.791, \mathrm{PS}=0.816, \mathrm{p}<0.001$ ) but also with global cognitive measures such as MMSE-KC (OS=-0.704, PS=-0.718, $\mathrm{p}<0.001)$. OS and PS of the DCAP-IADL were also correlated with global severity measures such as $\mathrm{CDR}(\mathrm{OS}=0.612, \mathrm{PS}=0.631, \mathrm{p}<$ 0.001). The AUC of OS and PS for dementia were 0.931 (95\% $\mathrm{CI}=0.900-0.963)$ and 0.940 (95\% CI=0.912-0.969), respectively (Table 5). The optimal cut-off point for dementia was $7 / 8$ (sensitivity $=0.902$, specificity $=0.837$ ) in OS and 6/7 (sensitivity $=0.902$, specificity $=0.841$ ) in PS, indicating that both OS and PS may have excellent diagnostic accuracy for dementia. ${ }^{34}$ The AUC of OS and PS for dementia were comparable to that of the K-IADL (AUC=0.921, 95\% CI=0.8930.949) and MMSE-KC (AUC $=0.919,95 \% \mathrm{CI}=0.892-0.946)$ (Figure 2).

Table 3. Mean scores of DCAP-IADL, K-IALD, BDS-ADL, and MMSE-KC in non-demented control subjects and dementia patients

\begin{tabular}{lcccc}
\hline & Non-demented control subjects & Dementia patients & $\mathrm{F}$ & $\mathrm{p}^{*}$ \\
\hline DCAP-IADL observed score & $4.40 \pm 4.02$ & $17.76 \pm 7.41$ & 568.36 & $<0.0001$ \\
DCAP-IADL predicted score & $3.57 \pm 3.78$ & $17.40 \pm 7.64$ & 641.43 & $<0.0001$ \\
K-IADL & $11.16 \pm 3.76$ & $21.94 \pm 6.65$ & 414.90 & $<0.0001$ \\
BDS-ADL & $0.51 \pm 0.58$ & $4.57 \pm 6.24$ & 806.04 & $<0.0001$ \\
MMSE-KC & $23.02 \pm 4.89$ & $12.74 \pm 5.02$ & 361.26 & $<0.0001$ \\
\hline
\end{tabular}

*mean scores of dementia patients are significantly different from non-dementia subjects after controlling for age, sex and education level by ANCOVA. DCAP-IADL: Dementia Care Assessment Packet-Instrumental Activities of Daily Living, K-IADL: Korean Instrumental Activities of Daily Living, BDS-ADL: Blessed Dementia Scale-Activities of Daily Living, ANCOVA: analysis of covariance, MMSE-KC: the Korean MMSE version of the Consortium to Establish a Registry for Alzheimer's Disease

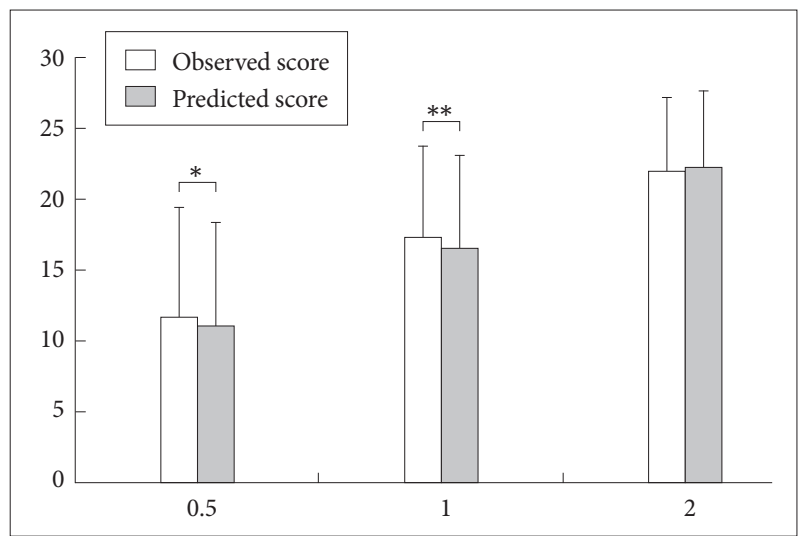

Figure 1. Mean scores on the Dementia Care Assessment Packet Instrumental Activities of Daily Living (DCAP-IADL) for the 3 different dementia stages according to CDR severity indices. All mean scores of the observed score and predicted score of DCAP-IADL differed significantly from one another by post hoc analysis (Bonferroni comparison, $p<0.05$ ). ${ }^{*} p=0.013$ by paired $t-$ test, ${ }^{*} p=0.001$ by paired t-test. CDR: Clinical Dementia Rating scale.
Table 4. Correlation between the Dementia Care Assessment Packet Instrumental Activities of Daily Living (DCAP-IADL) and other instruments for evaluating activities of daily living, global cognition and global severity of dementia

\begin{tabular}{lcc}
\hline & $\begin{array}{c}\text { DCAP-IADL } \\
\text { observed score }\end{array}$ & $\begin{array}{c}\text { DCAP-IADL } \\
\text { predicted score }\end{array}$ \\
\hline K-IADL & 0.840 & 0.845 \\
BDS-ADL & 0.784 & 0.815 \\
MMSE-KC & -0.704 & -0.718 \\
CDR & 0.612 & 0.631 \\
CDR-SOB & 0.791 & 0.816 \\
\hline
\end{tabular}

All correlations are significant at $\mathrm{p}<0.01$, by Pearson correlation (K-IADL, BDS-ADL, MMSE-KC, and CDR-SOB) and Spearman correlation analysis (CDR). K-IADL: Korean Instrumental Activities of Daily Living, BDS-ADL: Blessed Dementia Scale-Activities of Daily Living, MMSE-KC: the Korean MMSE version of the Consortium to Establish a Registry for Alzheimer's Disease, CDR: Clinical Dementia Rating scale, CDR-SOB: Clinical Dementia Rating scale-Sum of Box 
Table 5. Sensitivity and specificity of the Dementia Care Assessment Packet Instrumental Activities of Daily Living (DCAP-IADL) for diagnosis of dementia patients

\begin{tabular}{ccccc}
\hline Test & Cut-off & Sensitivity & Specificity & AUC \\
\hline DCAP-IADL observed score & & & 0.931 \\
$4 / 5$ & 0.955 & 0.654 & \\
$5 / 6$ & 0.938 & 0.742 & \\
$6 / 7$ & 0.920 & 0.808 & \\
$7 / 8^{*}$ & $0.902^{*}$ & $0.837^{*}$ & \\
$8 / 9$ & 0.884 & 0.864 & \\
$9 / 10$ & 0.866 & 0.897 & \\
DCAP-IADL predicted score & & & 0.940 \\
$4 / 5$ & 0.964 & 0.731 & \\
$5 / 6$ & 0.929 & 0.802 & \\
$6 / 7^{*}$ & $0.902^{*}$ & $0.841^{*}$ & \\
$7 / 8$ & 0.884 & 0.866 & \\
$8 / 9$ & 0.875 & 0.809 & \\
$9 / 10$ & 0.848 & 0.918 & \\
\hline
\end{tabular}

*optimal cut-off point as determined by ROC analysis. AUC: Area Under Curve, ROC: Receiver Operating Characteristics

\section{DISCUSSION}

In the present study, the DCAP-IADL was found to be a reliable and valid instrument for evaluating functional abilities of the elderly. A noteworthy result was that the reliability and validity of PS were comparable to or slightly better than those of OS. Most informant-based IADL instruments include some items that are not equally applicable to both genders in some cultures or not applicable in certain life styles. For example, in general, men do not prepare meals and women do not handle finances in many Asian cultures. Elders who lived with their children or dwell in nursing homes may not perform housekeeping duties, laundry, and food preparation by themselves. These items that subjects do not normally experience were not rated or given a minimum score in most previous IADL instruments. However, the DCAP-IADL excluded or minimized items that could be influenced by gender, culture, dwelling environment and life style. Moreover, to evaluate items that subjects have not experienced within an observation window, we used PS simultaneously with OS instead of excluding that item from the overall score or giving it a minimum score. In the PS of the DCAP-IADL, we asked informants to make their best guesses for the IADL items that could not be directly observed. Pre-existing tools did not adopt the scoring system like PS due to the concern of informant bias. However, our results suggest that the discriminant and concurrent validity of PS were comparable to or slightly better than those of OS. As Figure 1 shows, in comparison between OS and PS for the 3 different dementia stages according to CDR indices, OS

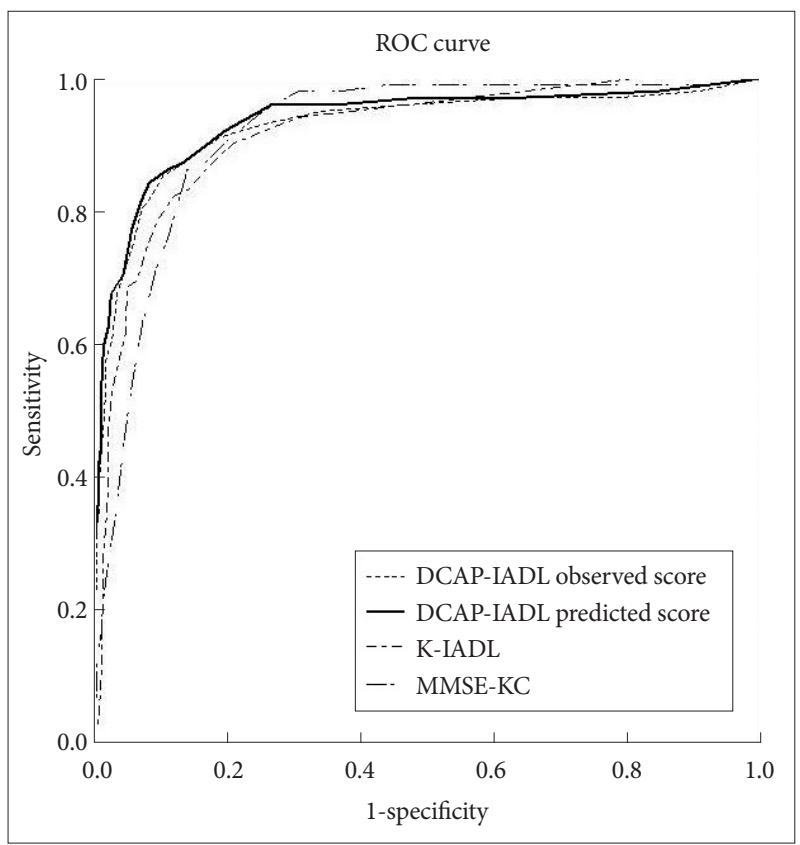

Figure 2. The area under curve (AUC) of DCAP-IADL observed score, DCAP-IADL predicted score, K-IADL, and MMSE-KC. DCAP-IADL: the Dementia Care Assessment Packet Instrumental Activities of Daily Living, K-IADL: Korean Instrumental Activities of Daily Living, MMSE-KC: the Korean MMSE version of the Consortium to Establish a Registry for Alzheimer's disease, ROC: Receiver Operating Characteristics.

was shown to be significantly higher than PS in very mild ( $\mathrm{p}=$ 0.013 , paired $\mathrm{t}$-test) and mild dementia $(\mathrm{p}=0.001$, paired $\mathrm{t}$ test). On the other hand, in more than moderate dementia, no difference was observed between OS and PS ( $\mathrm{p}=0.208$, paired t-test). These results suggest that OS might rate impairment of IADL in dementia patients much more severely than PS in early stage dementia. The reason for this discrepancy between OS and PS might be that the measurement of IADL was more influenced by the characteristics of subjects in early stage dementia. Therefore, PS may better reflect realistic IADL of dementia patients than OS especially those in early stage dementia. By minimizing the items that might be influenced by the characteristics of subjects (gender, culture, lifestyle and so on) and rating the items that were not actually performed in their everyday lives although they could performed such tasks in required situations, it is expected that inter-individual and intra-individual comparisons are easier and consistent in the evaluation of IADL. Except Lawton and Broody scale, ${ }^{5}$ the DCAP-IADL is a tool containing questionnaires only for IADL. Most previous tools such as the Alzheimer's Disease Cooperative Study-Activities of Daily Living scale (ADCSADL) ${ }^{35}$ BDS-ADL, ${ }^{28}$ the Bristol Activities of Daily Living scale (Bristol ADL), ${ }^{36,37}$ the Cleveland Scale for Activities of Daily Living (CSADL), ${ }^{38}$ the Disability Assessment for Dementia scale (DAD) $)^{39,40}$ and Interview for Deterioration of Daily living in Dementia (IDDD) $)^{41,42}$ include items probing 
both ADL and IADL. Even Alzheimer's Disease Cooperative Study-Activities of Daily Living-Prevention Instrument (ADCS-ADL-PI) ${ }^{43}$ include items for checking memory (ability to remember important dates and times, such as appointments or meetings), vision, hearing and mobility (ability to walk two blocks or further without help). Inclusion of items not related to IADL might cause unnecessary overlap of evaluation and difficulties in interpreting the results. To evaluate IADL solely, the DCAP-IADL excluded the items that were relatively unrelated to IADL (memory, comprehension and social functioning) and related to BADL. Nevertheless, our results showed that the DCAP-IADL (both OS and PS) have excellent diagnostic accuracy for dementia and is able to efficiently discriminate 3 different dementia stages. Barberger-Gateau et al. suggested that four IADL items (telephone use, use of transportation, responsibility for own medication, and handling money) are correlated with cognitive impairment independent of age, sex, and education. ${ }^{13}$ Furthermore, one year follow-up showed that the odds ratio for incident dementia, adjusted for age, increased from 10.6 for subjects dependent on one IADL to 318.4 for those dependent on four IADLs, compared with independent subjects. ${ }^{14}$ The baseline score of four IADLs was also a predictor of 3-year but not of 5-year incident dementia. ${ }^{15}$ For the purpose of screening dementia, global cognition tests, such as MMSE, are universally used. Although the DCAP-IADL was not originally designed as a screening instrument for dementia, the AUC of OS and PS for dementia are 0.931 (95\% CI=0.900-0.963) and 0.940 (95\% $\mathrm{CI}=0.912-0.969)$, respectively. These are comparable to and slightly higher than the AUC of MMSE-KC (AUC $=0.919$, 95\% CI=0.892-0.946), in spite of no significant difference. This suggests that the DCAP-IADL may be a useful tool for screening dementia. Moreover, evaluation of IADL by the DCAP-IADL is relatively simple and less time-consuming which makes this a clinically more feasible tool. IADL is important not only for evaluating dementia, but also for evaluating mild cognitive impairment (MCI). $\mathrm{MCI}$ is considered to be a prodromal stage of Alzheimer's disease (AD) and other forms of dementia. ${ }^{4-46}$ Petersen et al. ${ }^{47}$ proposed the following diagnostic criteria for MCI: 1) Not normal, not demented; 2) self and/or informant report and impairment on objective cognitive tasks; 3) Evidence of decline over time on objective cognitive tasks; and 4) Preserved basic activities of daily living/ Minimal impairment in complex instrumental functions. However, these criteria have not been applied uniformly across many studies ${ }^{47,48}$ due to variations in utilizing the diagnostic criteria. 49,50 "Minimal impairment in complex instrumental functions" among the diagnostic criteria was also an ambiguous criteria because there was no definite guideline for minimal impairment in IADL. In the presented study, we suggest an optimal cut-off point of 7/8 (sensitivity $90 \%$, specificity 84\%) for OS of the DCAP-IADL and an optimal cut-off point of 6/7 (sensitivity $90 \%$, specificity $84 \%$ ) for PS of the DCAPIADL. We can use these cut-off points as guidelines for minimal impairment in IADL. That is, over 7 (OS) or over 6 (PS) can be defined as impaired IADL in the dementia level and 1 to 6 (OS) or 1 to 5 (PS) can be defined as minimally impaired IADL in the MCI level. However, the limitations of the present study warrant further studies on the DCAP-IADL. First, the demographic characteristics of the subjects were not equal between the non-demented controls and dementia patients. As mentioned earlier in the report, the dementia patients were older and less educated than the non-demented controls. Women were more common in the dementia group than in the nondemented control group. These differences might influence on our results even though influence might be minimum level. Second, various dementia patients (Alzheimer's disease, vascular dementia, Dementia of Lewy body disease, Parkinson's disease dementia and so on) were enrolled in the present study. Diversity of dementia subjects might also influence on our results because physical disability in early stage vascular dementia or Parkinson's disease dementia, might have contributed to the DCAP-IADL scores rather than resulting in cognitive decline. Third, the present study was cross-sectional investigation. Thus, longitudinal studies on advanced dementia patients are needed to determine whether the DCAP-IADL can validly detect cognitive decline over time.

In conclusion, the DCAP-IADL is a reliable and valid instrument for evaluating the instrumental ability of daily living for the elderly, and may also be useful for screening dementia. Moreover, administering PS may enable the DCAP-IADL to overcome the differences in gender, culture and life style that hinder accurate evaluation in previous IADL instruments.

\section{Acknowledgments}

This study was supported by a grant from the Korean Health Technology R\&D Project, Ministry for Health, Welfare, \& Family Affairs, Republic of Korea (Grant No. A092077) and by the National Research Foundation of Korea (NRF) grant funded by the Korean government (MEST; Grant No. 2011-0018262). Also, this study was supported by an independent research grant from AstraZeneca Pharmaceuticals (Grant No. RC200811-399). The sponsors had no role in shaping the design, conduct, interpretation or summary of this research.

\section{REFERENCES}

1. American Psychiatric Association. Task Force on DSM-IV. Diagnostic and Statistical Manual of Mental Disorders: DSM-IV-TR. Washington, DC: American Psychiatric Association; 2000.

2. Desai AK, Grossberg GT, Sheth DN. Activities of daily living in patients with dementia: clinical relevance, methods of assessment and effects of treatment. CNS Drugs 2004;18:853-875.

3. Gauthier S, Gelinas I, Gauthier L. Functional disability in Alzheimer's disease. Int Psychogeriatr 1997;9(Suppl 1):163-165.

4. Dubois B, Feldman HH, Jacova C, Dekosky ST, Barberger-Gateau P, 
Cummings J, et al. Research criteria for the diagnosis of Alzheimer's disease: revising the NINCDS-ADRDA criteria. Lancet Neurol 2007; 6:734-746

5. Lawton MP, Brody EM. Assessment of older people: self-maintaining and instrumental activities of daily living. Gerontologist 1969;9:179186.

6. Peres K, Helmer C, Amieva H, Orgogozo JM, Rouch I, Dartigues JF, et al. Natural history of decline in instrumental activities of daily living performance over the 10 years preceding the clinical diagnosis of dementia: a prospective population-based study. J Am Geriatr Soc 2008; 56:37-44.

7. Wicklund AH, Johnson N, Rademaker A, Weitner BB, Weintraub S. Profiles of decline in activities of daily living in non-Alzheimer dementia. Alzheimer Dis Assoc Disord 2007;21:8-13.

8. Barberger-Gateau P, Alioum A, Peres K, Regnault A, Fabrigoule C, Nikulin $\mathrm{M}$, et al. The contribution of dementia to the disablement process and modifying factors. Dement Geriatr Cogn Disord 2004;18:330-337.

9. Njegovan V, Hing MM, Mitchell SL, Molnar FJ. The hierarchy of functional loss associated with cognitive decline in older persons. J Gerontol A Biol Sci Med Sci 2001;56:M638-M643.

10. De Lepeleire J, Heyman J, Buntinx F. The early diagnosis of dementia: triggers, early signs and luxating events. Fam Pract 1998;15:431-436.

11. Nygard L. Instrumental activities of daily living: a stepping-stone towards Alzheimer's disease diagnosis in subjects with mild cognitive impairment? Acta Neurol Scand Suppl 2003;179:42-46.

12. Oakley F, Sunderland T. Assessment of motor and process skills as a measure of IADL functioning in pharmacologic studies of people with Alzheimer's disease: a pilot study. Int Psychogeriatr 1997;9:197-206.

13. Barberger-Gateau P, Commenges D, Gagnon M, Letenneur L, Sauvel C, Dartigues JF. Instrumental activities of daily living as a screening tool for cognitive impairment and dementia in elderly community dwellers. J Am Geriatr Soc 1992;40:1129-1134.

14. Barberger-Gateau P, Dartigues JF, Letenneur L. Four Instrumental Activities of Daily Living Score as a predictor of one-year incident dementia. Age Ageing 1993;22:457-463.

15. Barberger-Gateau P, Fabrigoule C, Helmer C, Rouch I, Dartigues JF. Functional impairment in instrumental activities of daily living: an early clinical sign of dementia? J Am Geriatr Soc 1999;47:456-462.

16. Sikkes SA, de Lange-de Klerk ES, Pijnenburg YA, Scheltens P, Uitdehaag BM. A systematic review of Instrumental Activities of Daily Living scales in dementia: room for improvement. J Neurol Neurosurg Psychiatry 2009;80:7-12.

17. Graham DP, Kunik ME, Doody R, Snow AL. Self-reported awareness of performance in dementia. Brain Res Cogn Brain Res 2005;25:144152 .

18. Giovannetti T, Libon DJ, Hart T. Awareness of naturalistic action errors in dementia. J Int Neuropsychol Soc 2002;8:633-644.

19. Howorth P, Saper J. The dimensions of insight in people with dementia. Aging Ment Health 2003;7:113-122.

20. Onor ML, Trevisiol M, Negro C, Aguglia E. Different perception of cognitive impairment, behavioral disturbances, and functional disabilities between persons with mild cognitive impairment and mild Alzheimer's disease and their caregivers. Am J Alzheimers Dis Other Demen 2006;21:333-338.

21. Moore DJ, Palmer BW, Patterson TL, Jeste DV. A review of performance-based measures of functional living skills. J Psychiatr Res 2007; 41:97-118.

22. Feinstein AR, Josephy BR, Wells CK. Scientific and clinical problems in indexes of functional disability. Ann Intern Med 1986;105:413-420.

23. Won CW, Rho YG, SunWoo D, Lee YS. The validity and reliability of Korean Instrumental Activities of Daily Living (K-IADL) scale. J Korean Geriatr Soc 2002;6:273-280.

24. Kang SJ, Choi SH, Lee BH, Kwon JC, Na DL, Han SH, et al. The reliability and validity of the Korean Instrumental Activities of Daily Living (K-IADL). J Korean Neurol Assoc 2002;20:8-14.
25. Ku HM, Kim JH, Kwon EJ, Kim SH, Lee HS, Ko HJ, et al. A study on the reliability and validity of Seoul-Instrumental Activities of Daily Living (S-IADL). J Korean Neuropsychiatr Assoc 2004;43:189-199.

26. Kim KW, Woo JI, Yoon JC, Lee DY, Lee JH, Jhoo JH. Dementia Care Assessment Packet (DCAP). Seoul: The Korean Association for Dementia; 2002.

27. Jeong JW, Kim KW, Lee DY, Lee SB, Park JH, Choi EA, et al. A normative study of the Revised Hasegawa Dementia Scale: comparison of demographic influences between the Revised Hasegawa Dementia Scale and the Mini-Mental Status Examination. Dement Geriatr Cogn Disord 2007;24:288-293.

28. Lee JH, Lee KU, Lee DY, Kim KW, Jhoo JH, Kim JH, et al. Development of the Korean version of the Consortium to Establish a Registry for Alzheimer's Disease Assessment Packet (CERAD-K): clinical and neuropsychological assessment batteries. J Gerontol B Psychol Sci Soc Sci 2002;57:P47-P53.

29. American Psychiatric Association. Diagnostic and Statistical Manual of Mental Disorders: DSM-IV. Washington, DC: American Psychiatric Association; 1994.

30. Won CW, Rho YG, Kim SY, Lee E, Yoon JL, Cho KH, et al. The development of Korean Activities of Daily Liv ing (K-ADL) and Korean Instrumental Activities of Daily Living (K-IADL) Scale. Geriatrics 2002; 6:146-154.

31. Morris JC. Clinical dementia rating: a reliable and valid diagnostic and staging measure for dementia of the Alzheimer type. Int Psychogeriatr 1997;9(Suppl 1):173-176.

32. DeLong ER, DeLong DM, Clarke-Pearson DL. Comparing the areas under two or more correlated receiver operating characteristic curves: a nonparametric approach. Biometrics 1988;44:837-845.

33. Nunnally JC. Psychometric Theroy. New York: McGraw-Hill; 1978.

34. Metz CE. Basic principles of ROC analysis. Semin Nucl Med 1978;8: 283-298.

35. Galasko D, Bennett D, Sano M, Ernesto C, Thomas R, Grundman M, et al. An inventory to assess activities of daily living for clinical trials in Alzheimer's disease. The Alzheimer's Disease Cooperative Study. Alzheimer Dis Assoc Disord 1997;11(Suppl 2):S33-S39.

36. Bucks RS, Ashworth DL, Wilcock GK, Siegfried K. Assessment of activities of daily living in dementia: development of the Bristol Activities of Daily Living Scale. Age Ageing 1996;25:113-120.

37. Byrne LM, Wilson PM, Bucks RS, Hughes AO, Wilcock GK. The sensitivity to change over time of the Bristol Activities of Daily Living Scale in Alzheimer's disease. Int J Geriatr Psychiatry 2000;15:656-661.

38. Patterson MB, Mack JL, Neundorfer MM, Martin RJ, Smyth KA, Whitehouse PJ. Assessment of functional ability in Alzheimer disease: a review and a preliminary report on the Cleveland Scale for Activities of Daily Living. Alzheimer Dis Assoc Disord 1992;6:145-163.

39. Gelinas I, Gauthier L, McIntyre M, Gauthier S. Development of a functional measure for persons with Alzheimer's disease: the disability assessment for dementia. Am J Occup Ther 1999;53:471-481.

40. Feldman H, Sauter A, Donald A, Gelinas I, Gauthier S, Torfs K, et al. The disability assessment for dementia scale: a 12-month study of functional ability in mild to moderate severity Alzheimer disease. Alzheimer Dis Assoc Disord 2001;15:89-95.

41. Teunisse S, Derix MM. Measurement of activities of daily living in patients with dementia living at home: development of a questionnaire. Tijdschr Gerontol Geriatr 1991;22:53-59.

42. Bohm P, Pena-Casanova J, Aguilar M, Hernandez G, Sol JM, Blesa R. Clinical validity and utility of the interview for deterioration of daily living in dementia for Spanish-speaking communities NORMACODEM Group. Int Psychogeriatr 1998;10:261-270.

43. Galasko D, Bennett DA, Sano M, Marson D, Kaye J, Edland SD, et al. ADCS Prevention Instrument Project: assessment of instrumental activities of daily living for community-dwelling elderly individuals in dementia prevention clinical trials. Alzheimer Dis Assoc Disord 2006; 20(4 Suppl 3):S152-S169. 
44. Petersen RC, Thomas RG, Grundman M, Bennett D, Doody R, Ferris S, et al. Vitamin $\mathrm{E}$ and donepezil for the treatment of mild cognitive impairment. N Engl J Med 2005;352:2379-2388.

45. Morris JC, Storandt M, Miller JP, McKeel DW, Price JL, Rubin EH, et al. Mild cognitive impairment represents early-stage Alzheimer disease. Arch Neurol 2001;58:397-405.

46. Petersen RC, Smith GE, Waring SC, Ivnik RJ, Tangalos EG, Kokmen E. Mild cognitive impairment: clinical characterization and outcome. Arch Neurol 1999;56:303-308.

47. Winblad B, Palmer K, Kivipelto M, Jelic V, Fratiglioni L, Wahlund LO, et al. Mild cognitive impairment--beyond controversies, towards a con- sensus: report of the International Working Group on Mild Cognitive Impairment. J Intern Med 2004;256:240-246.

48. Petersen RC. Mild cognitive impairment as a diagnostic entity. J Intern Med 2004;256:183-194.

49. Busse A, Bischkopf J, Riedel-Heller SG, Angermeyer MC. Mild cognitive impairment: prevalence and incidence according to different diagnostic criteria. Results of the Leipzig Longitudinal Study of the Aged (LEILA75+). Br J Psychiatry 2003;182:449-454.

50. Petersen RC, Doody R, Kurz A, Mohs RC, Morris JC, Rabins PV, et al. Current concepts in mild cognitive impairment. Arch Neurol 2001;58: 1985-1992. 